\title{
A Multi-class BCI based on Somatosensory Imagery
}

\author{
Lin Yao, Natalie Mrachacz-Kersting, Xinjun Sheng, \\ Xiangyang Zhu, Dario Farina, Fellow, IEEE, Ning Jiang, Senior Member, IEEE
}

\begin{abstract}
In this study, we investigated the performance of a multi-class brain-computer interface (BCI). The BCI system is based on the concept of somatosensory attentional orientation (SAO), in which the user shifts and maintains somatosensory attention by imagining the sensation of tactile stimulation of a body part. At the beginning of every trial, a vibration stimulus $(200 \mathrm{~ms})$ informed the subjects to prepare for the task. Four SAO tasks were performed following randomly presented cues: SAO of the left hand (SAO-LF), SAO of the right hand (SAO-RT), bilateral SAO (SAO-BI), and SAO suppressed or idle state (SAOID). Analysis of the event-related desynchronization and synchronization (ERD/ERS) in the EEG indicated that the four SAO tasks had different somatosensory cortical activation patterns. SAO-LF and SAO-RT exhibited stronger contralateral ERD, whereas bilateral ERD activation was indicative of SAO-BI, and bilateral ERS activation was associated with SAO-ID. By selecting the frequency bands and/or optimal classes, classification accuracy of the system reached $85.2 \pm 11.2 \%$ for two classes, $\mathbf{6 9 . 5} \pm \mathbf{1 6 . 2 \%}$ for three classes, and $55.9 \pm 15.8 \%$ for four classes. The results validated a multi-class BCI system based on SAO, on a single trial basis. Somatosensory attention to different body parts induces diverse oscillatory dynamics within the somatosensory area of the brain, and the proposed SAO paradigm provided a new approach for a multiple-class BCI that is potentially stimulusindependent.
\end{abstract}

Index Terms - Brain computer interface (BCI), Somatosensory Attentional Orientation (SAO), Somatosensory Imagery, Oscillatory Dynamics, Multi-class BCI

\section{INTRODUCTION}

Brain-computer interface (BCI) systems provide an alternative non-muscular control and communication channel for users with severe disabilities to interact with the external world [1]. Brain signals are collected either invasively [2] or non-invasively [3], [4] while the user performs mental tasks. Time and/or frequency domain features are extracted and classified for external device control [5]. BCIs have been successfully implemented in a wide variety of neuromuscular disorders [6]-[10], both for communication (e.g. amyotrophic lateral sclerosis - ALS) and for restoration of impaired motor function (e.g. stroke). In addition, they have shown viability in controlling applications such as wheelchair command [11], [12], helicopter navigation [13], [14], robotic arm control [15], and in neurorehabilitation [16]-[19].

Motor imagery (MI) is one of the most commonly investigated mental tasks in BCIs research [20]-[22]. MI is independent of external stimuli and has shown a therapeutic

Lin Yao and Ning Jiang are with Department of Systems Design Engineering, Faculty of Engineering, University of Waterloo, Waterloo, Canada (Corresponding E-mail: ning.jiang@uwaterloo.ca).

Xinjun Sheng and Xiangyang Zhu are with State Key Lab of Mechanical System and Vibration, Shanghai Jiao Tong University, Shanghai, China.

Natalie Mrachacz-Kersting is with Center for Sensory-Motor Interaction, the Faculty of Medicine, Aalborg University, Aalborg, Denmark.

Dario Farina is with Department of Bioengineering, Imperial College London, London, UK. benefit in neurorehabilitation [1], [23], [24]. MI (e.g. of the left or right hand) [25] induces event-related desynchronization/synchronization (ERD/ERS) often referred to as sensory-motor rhythms (SMR) [26]-[29]. Left hand MI results in a contralateral right hemisphere ERD and ipsilateral left hemisphere ERS, while right hand MI results in a left hemisphere ERD and right hemisphere ERS [30]. The ERD/ERS dynamic can not only be elicited by imagined or real movement, but also correlates strongly with the human somatosensory system in the processing of afferent inflow [31]. The oscillatory rhythmic activity induced by sensory stimulation should reflect how the brain processes these stimuli, e.g. tactile stimuli [32], [33]. In a recent series of studies [34][38], we have demonstrated that a tactile selective sensation (SS) BCI based on stimulus-induced oscillatory dynamics outperforms other types of tactile-based BCIs, such as those based on steady-state somatosensory evoked potentials (SSSEPs) [39]-[41]. Further, we also demonstrated that a topdown somatosensory attention shift (e.g. on either left or right hand) can be reliably detected from the spontaneous EEG rhythms, even without the presence of the actual stimuli. We termed this sensory imagery (SI) task "somatosensory attentional orientation" (SAO). We also recently combined MI with SAO to improve conventional MI-based BCI performance and increase the number of commands [42].

MI and SI are both based on the oscillatory dynamics of brain activity quantified as ERD/ERS, and both are imagined, thus covert mental tasks. Specifically, in SAO tasks, subjects shift and maintain their somatosensory attention to different body parts (e.g. left or right hand), and imagine a sensation of tactile

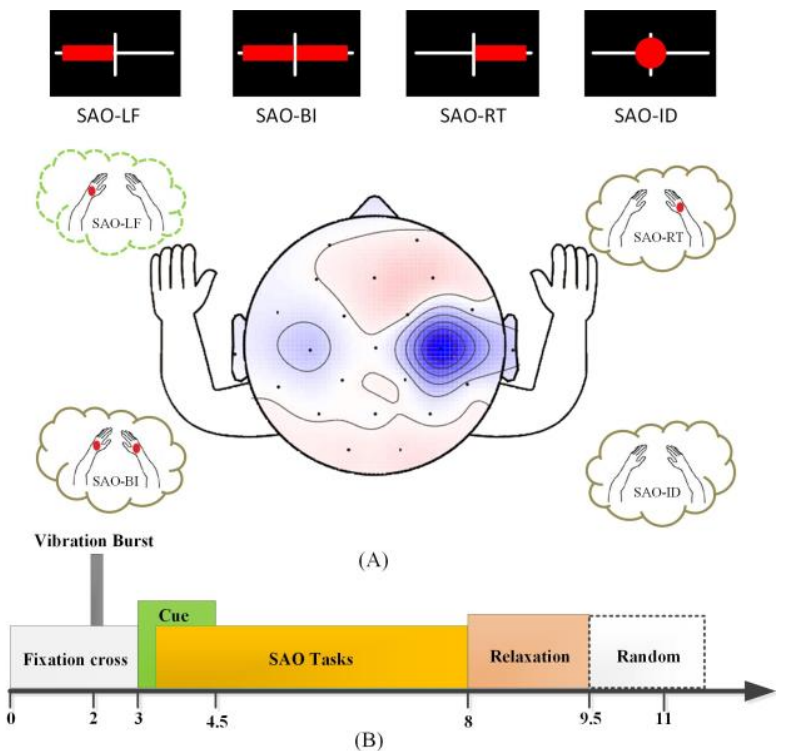

Figure 1. Illustration of the experimental protocol. (A) Graphic illustration of the SAO tasks (SAO-LF, SAO-RT, SAO-BI, SAO-ID). (B) The temporal sequence of each trial in SAO paradigm. 


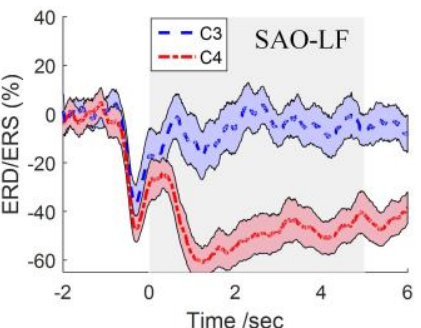

(A)

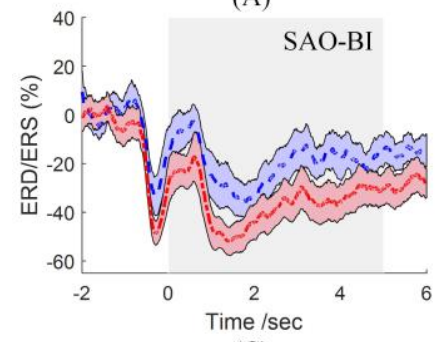

(C)

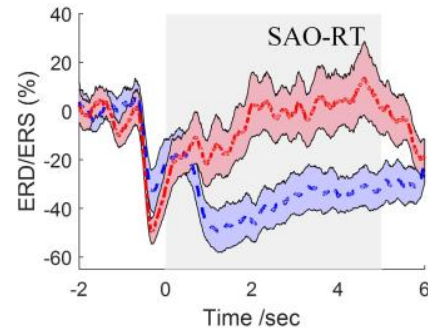

(B)

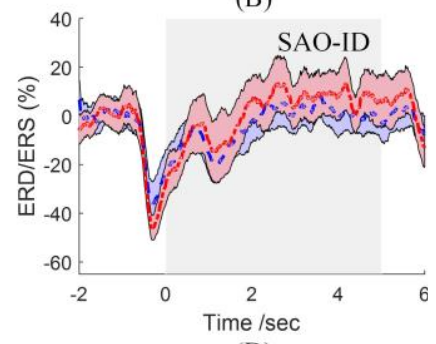

(D)

Figure 2. The grand-averaged ERD/ERS dynamics at small-Laplace filtered $\mathrm{C} 3$ and $\mathrm{C} 4$ channels within [8 26] $\mathrm{Hz}$ (alpha-beta band). ERD/ERS corresponds to SAO-LF task in (A), SAO-RT task in (B), SAO-BI task in (C) and SAO-ID task in (D). The upper and lower curves indicate standard error. Note: time 0s corresponds to the start of the task.

vibrational stimulus being applied. The resulting EEG signals can be classified with an accuracy $>75 \%$ for two classes [37], [42]. In the current study, we aimed to extend the current SAO based two-class BCI to a multiple-class BCI system in order to provide a new approach to expand the currently limited BCI commands.

Tactile stimulus-induced oscillatory dynamics have been shown to encode the subjects' covert somatosensory attention in a multi-class tactile BCI [38]. Decoding these led to a promising tactile $\mathrm{BCI}$ performance as compared with the existing modalities of tactile BCIs. Furthermore, this SAO modality, where actual tactile stimuli are absent, had a reliable BCI performance when left and right SAO tasks were investigated [37], [43]. This motivated the current study design where subjects were instructed to perform four SAO tasks: 1) SAO of the left hand (SAO-LF); 2) SAO of the right hand (SAO-RT); 3) Bilateral SAO (SAO-BI); 4) SAO suppressed or idle (SAO-ID). In order to investigate only the BCI performance of the task effect, no feedback was provided to the subjects in order to minimize subjective adaptation. The

oscillatory dynamics accompanying somatosensory attention to sensation of different body parts were investigated and the feasibility of a SAO-based multiple-class BCI system was systematically evaluated.

\section{METHODOLOGY}

\section{A. Subjects and EEG recording}

A total of 19 healthy subjects participated in the experiments (10 females, all right-handed, average age 21.8 \pm 2.5 years). All subjects were BCI naïve. The study was reviewed and approved by the Ethics Committee of the University of Waterloo, Waterloo, Canada (ORE\#: 22295). Before participation, all participants signed the informed consent forms.

A wireless g.Nautilus EEG system with 32 channels (gtec, Austria) was used to record EEG signals. Electrodes were placed in accordance with the extended 10/20 system. The reference electrode was placed on the right earlobe, and the ground electrode was located on the forehead. The signals were digitally sampled at $250 \mathrm{~Hz}$. Linear resonant actuators $(10 \mathrm{~mm}$, C10-100, Precision Microdrives Ltd., typical normalized amplitude $1.4 \mathrm{G}$ ) were used for producing vibrotactile stimulation [43].

\section{B. Experimental Protocol}

Before starting the experiment, subjects were explicitly instructed not to perform any attempt or imagined movement; they were asked to only focus, according to cues shown, on the sensation in their left hand, right hand, both, or none. For the SAO tasks, the subjects were instructed to concentrate their somatosensory attention on either the left or the right wrist, and to imagine that a tactile stimulus (vibration) was being applied to these. Subjects were asked to continue imagining the tactile sensation while maintaining their attention on the corresponding hand. Four SAO tasks were randomly performed, according to the cue: 1) SAO-LF; 2) SAO-RT; 3) SAO-BI; 4) SAO-ID. The experimental paradigm is depicted in Fig. 1(A)(B). The subjects were asked to minimize eye blinks and body movements, and to avoid muscle contraction during the mental tasks. At the beginning of each trial $(\mathrm{T}=0 \mathrm{~s})$, a white fixation cross ("+") was presented in the center of the screen.

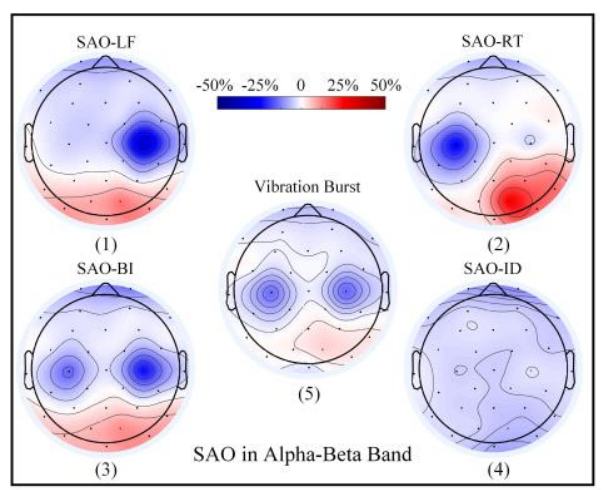

(A)

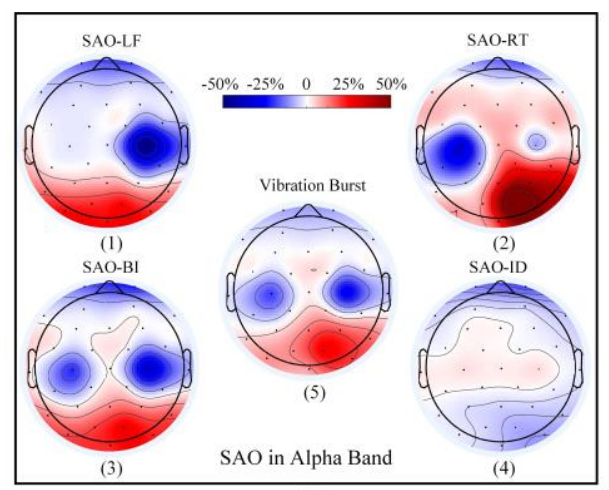

(B)

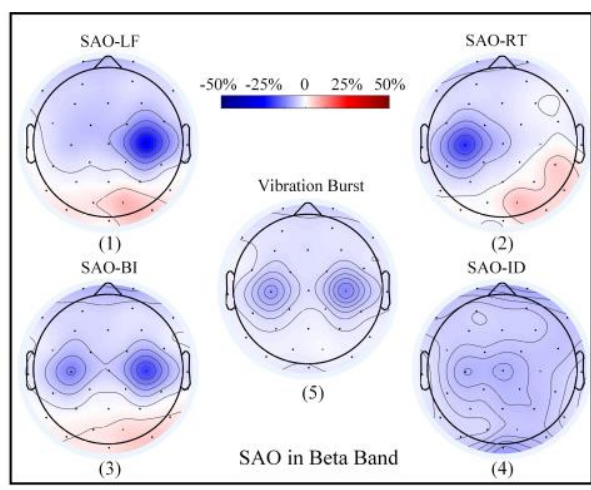

(C)

Figure 3. Grand-averaged ERD/ERS distribution within [8 26] Hz alpha-beta band in (A), [8 13] alpha band in (B) and [13 26] beta band in (C). (A)(1) ERD/ERS distribution with respect to SAO-LF task. (A)(2) ERD/ERS distribution with respect to SAO-RT task. (A)(3) ERD/ERS distribution with respect to SAO-BI task. (A)(4) ERD/ERS distribution with respect to SAO-ID task. (A)(5) ERD/ERS distribution with respect to vibration burst at the beginning of the trial. The subfigures in the panel $(B)$ and $(C)$ was similar as in panel (A). 


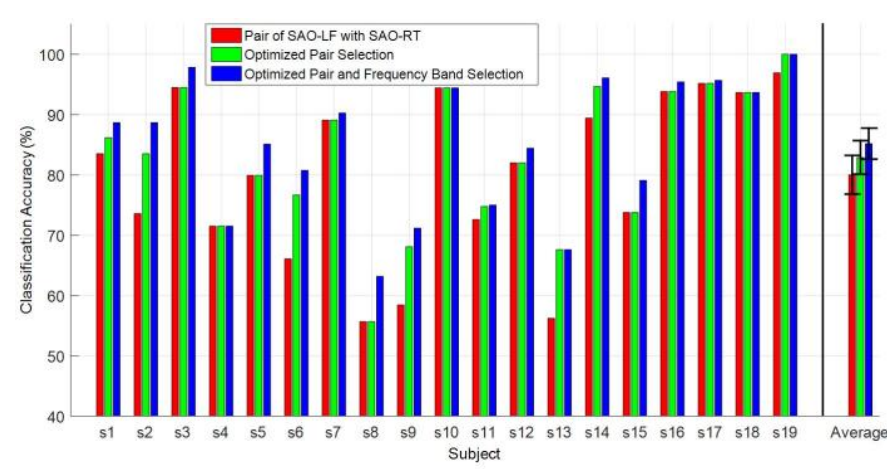

Figure 4. Classification accuracy in two-class BCI systems. The red bars indicate the BCI performance based on SAO-LF and SAO-RT, which we proposed in [35], [40]; the green bars the BCI performance with the optimal task pair for each subject; the blue bars the BCI performance with both task pair and frequency band optimization. The error bars represent the standard error, with $\mathrm{N}=19$ (number of the subjects). The chance level classification is $61.67 \%$.

At $\mathrm{T}=2 \mathrm{~s}$, a 200-ms vibration pulse was applied to both hands to alert the subjects of the subsequent task. At $\mathrm{T}=3 \mathrm{~s}$, a red visual cue appeared for $1.5 \mathrm{~s}$. When the cue was a left-pointing arrow, it indicated the SAO-LF task; a right-pointing arrow the SAO-RT task; a double-sided arrow the SAO-BI task; and a circle the SAO-ID task. The mental task lasted $5 \mathrm{~s}$, until the fixation symbol disappeared ( $\mathrm{T}=8 \mathrm{~s})$. Finally, subjects were instructed to relax for a random time interval of $1.5 \sim 3.5 \mathrm{~s}$, so that the subject would not be habituated. A total of 240 trials were performed by the subject in six runs, with 10 trials of each task in each run in random order. There was a 2-4 min break between consecutive runs.

\section{Data analysis}

A fourth order Butterworth filter of [8 26] Hz was utilized for EEG filtering prior to ERD/ERS calculation. ERD (ERS) is defined as the percentage of band power decrease (increase) with respect to a reference interval (e.g. resting state before the task). The reference interval from 2.0 to $1.2 \mathrm{~s}$ prior to the

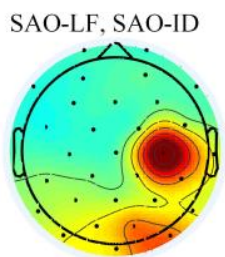

(1)

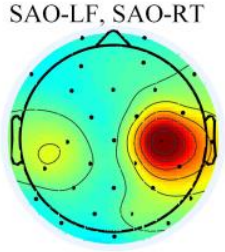

(4)

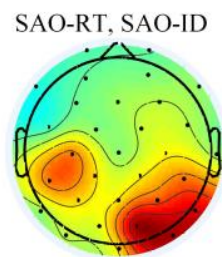

(2)

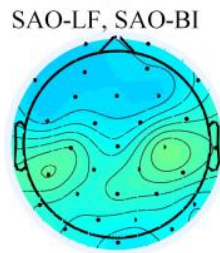

(5)

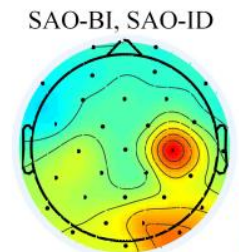

(3)

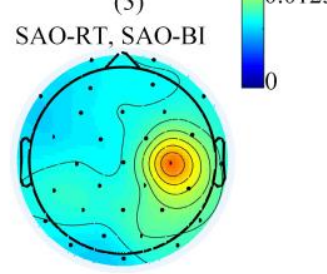

(6)
Figure 5. Grand-averaged $R^{2}$ among different two-class BCI modalities within $[826] \mathrm{Hz}$ frequency band. (1) $R^{2}$ discriminative information distribution between SAO-LF and SAO-ID. (2) $\mathbf{R}^{2}$ discriminative information distribution between SAO-RT and SAO-ID. (3) $\mathbf{R}^{2}$ discriminative information distribution between SAO-BI and SAO-ID. (4) $R^{2}$ discriminative information distribution between SAO-LF and SAO-RT. (5) $\mathbf{R}^{2}$ discriminative information distribution between SAO-LF and SAOBI. (6) $\mathbf{R}^{2}$ discriminative information distribution between SAO-RT and SAO-BI. The color bar indicates the $R^{2}$ value.

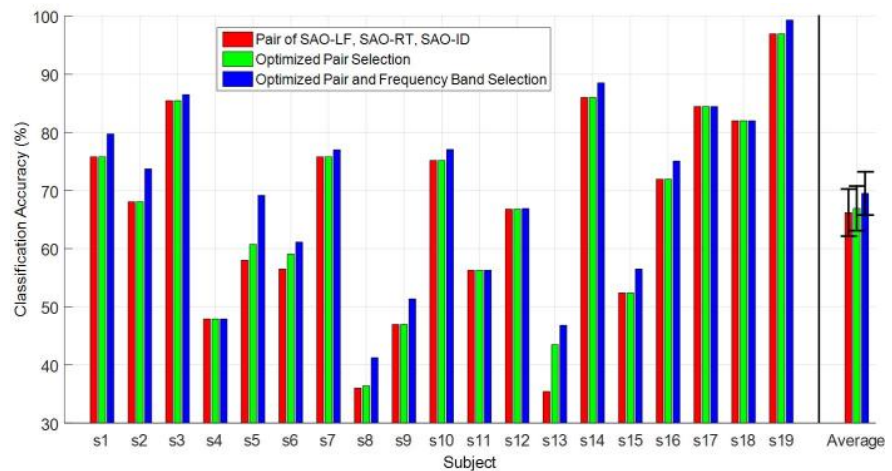

Figure 6. Classification accuracy in three-class BCI systems. Red bars indicate the BCI performance based on SAO-LF, SAO-RT and SAO-ID. Green bars indicate the BCI performance after selection of individual optimal task pair; the blue bars the BCI performance with both task pair and frequency band optimization. The error bars represent the standard error, with $N=19$ (number of the subjects). The chance level classification is $\mathbf{4 2 . 7 8 \%}$

appearance of the arrow (i.e. 1 to $1.8 \mathrm{~s}$ from the beginning of the trial) was used for ERD/ERS calculation. To determine the cortical activation dynamics involved in each mental task, the grand averages of ERD/ERS from all subjects within the same task were calculated.

Time-frequency decomposition of all the EEG channels was performed to construct the spatio-spectral-temporal structure within each SAO tasks. It was calculated every $200 \mathrm{~ms}$ with a Hanning tapper, and the number of cycles per window was chosen as seven to achieve adequate frequency and time resolution [44]. The $\mathrm{R}^{2}$ index is defined as the squared Pearsoncorrelation coefficient between feature and class label [45], [46]. Based on the above spatio-spectral-temporal structures between different mental tasks, an $\mathrm{R}^{2}$ in the spatio-spectraltemporal space was formulated and used to interpret the classification results between different SAO tasks.

Spatial filtering based on Common Spatial Pattern (CSP) [47], [48], was used for enhancing feature discrimination among the investigated somatosensory attention tasks. The feature vectors were comprised of the log-variance values of the first and last three CSP components. Linear discriminative analysis (LDA) was used for classification. Moreover, a $10 \times 10$ cross-validation was utilized for offline BCI performance evaluation, and for selecting the subject-specific frequency band. The frequency bands were individually selected for each subject among the following [37]: alpha-beta [8 26] Hz $(\alpha \beta)$, alpha [8 13] Hz ( $\alpha)$, beta [13 26] Hz ( $\beta)$, lower alpha [8 10] Hz $(\alpha-)$, upper alpha $\left[\begin{array}{ll}10 & 13\end{array}\right] \mathrm{Hz}(\alpha+)$, lower beta $[1326] \mathrm{Hz}(\beta-)$, upper beta [20 26] Hz $(\beta+)$, and eta [10 16] Hz (ๆ) [38].

The interval for EEG signal analysis and classification was chosen as $1 \mathrm{~s}$ to $4 \mathrm{~s}$ after the appearance of the cue bar (i.e. $4 \mathrm{~s}$ to $7 \mathrm{~s}$ after the beginning of the trial. For the 2-class classification, six class pairs were analyzed: SAO-LF vs SAORT (P1), SAO-LF vs SAO-BI (P2), SAO-LF vs SAO-ID (P3), SAO-RT vs SAO-BI (P4), SAO-RT vs SAO-ID (P5), and SAOBI vs SAO-ID (P6). Four 3-class scenarios were investigated: SAO-LF vs SAO-RT vs SAO-BI (T1), SAO-LF vs SAO-RT vs SAO-ID (T2), SAO-LF vs SAO-BI vs SAO-ID (T3), and SAORT vs SAO-BI vs SAO-ID (T4). Further, the four tasks were also classified in a 4-class scenario. To the general applicability of the SAO-based attention BCI system, we investigated the 


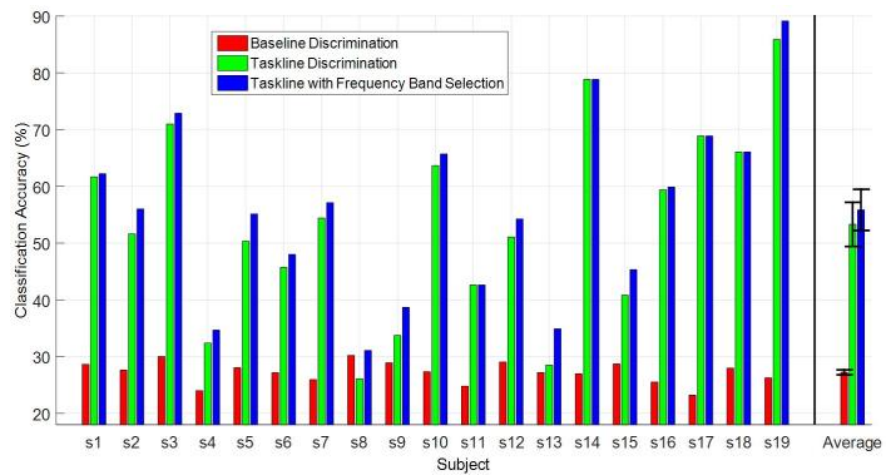

Figure 7. Classification accuracy in four-class BCI systems. The red bars and green bars represents the performance within the baseline time period and task period separately. Blue bars indicate the BCI performance after frequency band selection. The error bars represent the standard error, with $\mathrm{N}=19$ (number of the subjects). The chance level classification is $32.50 \%$. The base-line period was $[-20] \mathrm{s}$ before the appearance of the cue.

performance of the above 2-class, 3-class and 4-class conditions offline, within a fixed frequency band of [8 26] $\mathrm{Hz}(\alpha \beta)$ for all subjects. Moreover, we also performed optimization of frequency bands and task scenarios subject-specific, to explore the best performance for individual subjects due to the existence of a large inter-subject variability [49].

\section{Statistics}

Performance among different BCI task pairs was analyzed using a one-way ANOVA with repeated-measures $(p=0.05)$. Whenever the main effect was found to be significant, Bonferroni correction was used for post-hoc testing to identify which level(s) of the factor were statistically different from other levels. The theoretical chance level was corrected with the number of trials [50]. The upper limit of a $99 \%$ confidence interval for chance accuracy based on the number of trials in this study was $61.67 \%$ for two classes, $42.78 \%$ for three classes, and $32.50 \%$ for four classes.

\section{RESULTS}

\section{A. Oscillatory Dynamics of Somatosensory Attention to Imagined Body Sensation}

Fig. 2 shows the grand-averaged oscillatory dynamics within the alpha-beta frequency band across the four imagined sensation tasks. At $-1 \mathrm{~s}$, a 200 ms vibration burst induced a band power decrease that was similar in all trials in the channels $\mathrm{C} 3$ and $\mathrm{C} 4$ (C3 and $\mathrm{C} 4$ were chosen since they were located over the sensory-motor regions). The cortical activity (ERD) reached a maximum at $0.5 \mathrm{~s}$ after the vibration burst. The somatosensory activation induced by the vibration burst is also indicated in Fig. 3(A)(5), demonstrating a clear lateralization. From $0 \mathrm{~s}$ to $5 \mathrm{~s}$, subjects performed imagined sensation tasks when no sensory stimuli were applied. These resulted in diverse oscillatory activation mainly around both somatosensory cortex. During the SAO-LF task, the ERD on the right hemisphere was more pronounced; conversely, during the SAO-RT task, the ERD in the left hemisphere was more prominent. During SAO-BI, a clear bilateral activation was present and this was stronger when compared to that during the SAO-ID task, while during the
SAO-ID task, the ERD strength was much lower bilaterally compared to the other three tasks. ERD/ERS within the [8 26] $\mathrm{Hz}$ alpha-beta frequency band across the whole scalp are shown in Fig. 3(A).

Grand-averaged ERD/ERS distributions within the alpha and beta bands are shown in Fig. 3(B) and (C). The activation pattern in both alpha and beta bands were similar and topographically distributed with respect to the body schema. Further, the SAO-induced oscillatory change (ERD) and the occipital alpha ERS were both stronger within the alpha band.

\section{B. BCI Performance for Two-class Scenarios}

Using the [8 26] $\mathrm{Hz}$ frequency band for all subjects, the average accuracy was $80.0 \pm 14.0 \%$ for P1 (SAO-RT vs SAOLF), $66.8 \pm 13.0 \%$ for $\mathrm{P} 2,76.4 \pm 13.5 \%$ for $\mathrm{P} 3,70.9 \pm 11.8 \%$ for $\mathrm{P} 4,76.1 \pm 14.8 \%$ for $\mathrm{P} 5$, and $73.2 \pm 11.9 \%$ for P6. One-way ANOVA with repeated measure showed that there was a significant difference in classification accuracy among different pairs $\left(\mathrm{F}_{(5,90)}=12.32, p<0.01\right)$. Post-hoc testing $(p=0.05)$ revealed that P1 was significantly better than P2, P4 and P6, and no significant difference was found among P1, P3, and P5. When the best two-class task pair for each individual subject was used (optimized pair selection in Fig. 4), the average classification accuracy increased to $82.9 \pm 12.2 \%(p<0.01)$, with the best result on an individual subject being above $95 \%$. Moreover, when the subject-specific frequency band was selected, the average accuracy further increased to $85.2 \pm 11.2 \%$. Sixteen out of 19 subjects were above the corrected chance level of $61.67 \%$ when pair P1 and the frequency band of [8 26] Hz was selected. The number of subjects with a classification accuracy above chance level increased to 18 out of 19 when the optimized pair was selected, and all subjects surpassed the chance level when both optimized pair and frequency band were utilized. For a twoclass BCI control to be considered as usable, $70 \%$ accuracy has been defined as the threshold value in previous studies, i.e. lower than $70 \%$ is normally considered as BCI-illiterate [51], [52]. There are approximately $30 \%$ of subjects whose BCI performance is below the $70 \%$ accuracy level [53]. In the current study, 15 subjects crossed the $70 \%$ accuracy level, which equates to $79.0 \%$ of total number of subjects $(15 / 19)$. Conversely, this meant that the BCI-illiteracy rate of the proposed system was $21 \%(4 / 19)$. With task pair and subject optimal frequency band selection, the BCI-illiteracy rate further reduced to $10.5 \%$ (2/19). (Fig. 4).

The discriminative information distribution among different pairs, represented as $\mathrm{R}^{2}$, is shown in Fig. 5 (grand-average among subjects).

\section{BCI Performance on Three and Four Class Scenarios}

Using the frequency band of [8 26] Hz for all subjects, the average accuracy in the three-class scenarios was $58.7 \pm 12.2 \%$ for $\mathrm{T} 1,66.2 \pm 17.7 \%$ for $\mathrm{T} 2,58.7 \pm 15.1 \%$ for $\mathrm{T} 3$, and $60.7 \pm 15.6 \%$ for T4. One-way ANOVA with repeated measures revealed a significant difference in classification accuracy between the four scenarios $\left(\mathrm{F}_{(3,54)}=14.99, p<0.01\right)$, and post-hoc comparison $(p=0.05)$ showed that the performance of T2 was significantly better than the other three scenarios, and no significant difference was found between T1, T3 and T4. Fig. 6 illustrates the performance of the three-class BCI system. Seventeen out of 19 subjects exceeded the corrected random 


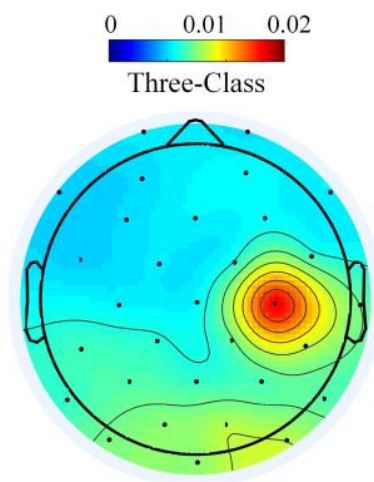

(1)

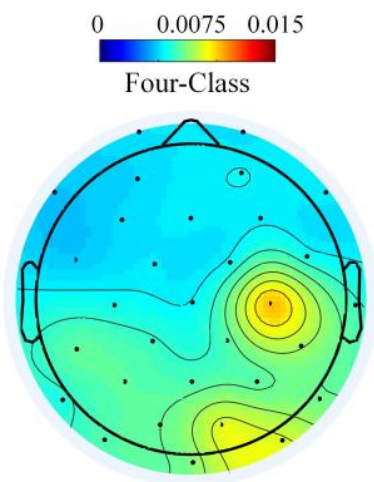

(2)
Figure 8 Grand-averaged $R^{2}$ value distribution from three-class and fourclass BCI modality within $\left[\begin{array}{ll}8 & 26\end{array}\right] \mathrm{Hz}$ frequency band. (1) $R^{2}$ discriminative information distribution among SAO-LF, SAO-RT and SAO-ID. (2) $\mathbf{R}^{2}$ discriminative information distribution among SAO-LF, SAO-RT, SAO$B I$ and SAO-ID. The color bar indicates the $R^{2}$ value.

chance level of $42.78 \%$. The selection of the optimal pair did not substantially improve the performance with respect to $\mathrm{T} 2$. However, subject-specific frequency band and task pair selection significantly improved the accuracy $(69.5 \pm 16.2 \%$, Paired-t test $p<0.05$ ).

Fig. 7 shows the performance of the four-class SAO based BCI system. The task activity discrimination accuracy was compared against the baseline performance, when no tasks were performed. The mean base-line accuracy was $27.2 \pm 1.9 \%$, which was expected for a four-class scenario ( $25 \%$ in theory). The performance achieved an average accuracy of $53.3 \pm 17.0 \%$, and 16 out of 19 subjects exceeded the corrected random chance level of $32.50 \%$. For this scenario, a performance increase of approximately $3 \%(p=0.05)$ was achieved when the subjectspecific frequency band was selected.

The discriminative information distribution with respect to the three-class and four-class BCI system, represented as $\mathrm{R}^{2}$, is shown in Fig. 8 (grand-average). The electrode $\mathrm{C} 4$ located over the right somatosensory cortex contributed the most to the classification results in the current experimental setting.

\section{DISCUSSION}

In this study the characteristics of oscillatory dynamics with respect to a type of SI task, i.e. SAO, were systematically investigated. The SAO task induced a clear somatosensoryattention induced oscillatory power decrease in both the alpha and beta frequency band when attention was shifted and maintained on the corresponding body part (left, right, and both hands) under the condition that no actual stimuli were applied. We also presented a novel approach for a multiple-class somatosensory BCI. To the best of our knowledge, this is the first time that a somatosensory attention based multiple-class stimulus-independent $\mathrm{BCI}$ has been proposed and experimentally validated. The SAO-based BCI differs from the typical motor imagery $\mathrm{BCI}$ because the extracted brain signal would be generated from the somatosensory cortex rather than mainly from the motor cortex. The three-class discrimination showed an average accuracy of $69.5 \pm 16.2 \%$ and the four-class discrimination $53.3 \pm 17.0 \%$. These are comparable performances with respect to the existing multiple-class MIbased BCI systems. Building on the pioneer studies of brain

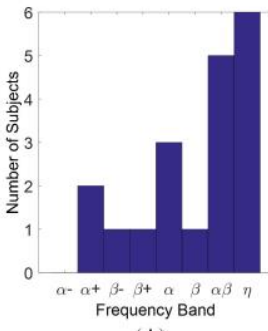

(A)

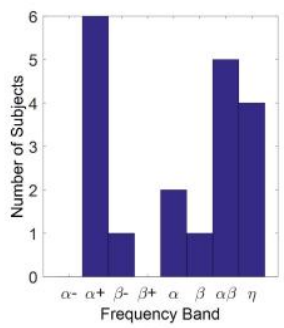

(B)

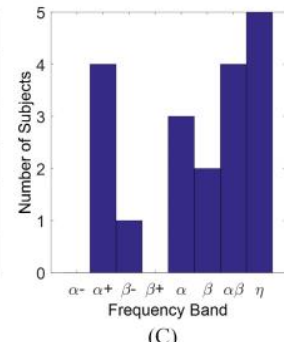

(C)
Figure 9. Number of subjects in selected optimal frequency bands. (A) corresponds to the subject number distribution in two-class BCI, i.e. SAOLF vs SAO-RT. (B) distribution in three-class BCI, i.e. SAO-LF vs SAORT vs SAO-ID. (C) distribution in four-class BCI.

activation with respect to MI on different body parts, such as left hand, right hand, foot, and tongue, a first four-class MI BCI system was first proposed by Pfurtscheller [54], demonstrating a mean kappa coefficient of 0.418 , which equates to a mean classification accuracy of $56.3 \%$. This four-class BCI system was fully explored through several BCI competitions to improve its performance by designing suitable machine learning algorithms [55], [56]. Besides MI, other mental tasks have been proposed to increase the number of classes and their discrimination. For example, Friedrich [57] discriminated word association, mental subtraction, and spatial navigation with a comparable performance as the four-class MI-based BCI. Moreover, the combination of MI tasks has been used, such as simultaneous imagination of both left and right hands movement [14]. Since SAO is able to selectively activate the somatosensory cortex, it offers a novel avenue to be combined with MI based BCIs, increasing the different command types that will expand the currently limited BCI output. In our previous experimental study of MI with SAO [42], we found that the hybrid modality with left SAO and right MI tasks significantly improved conventional left and right MI discriminability.

BCI systems based on left-hand MI and right-hand MI are the state-of-the-art of stimulus-independent BCIs. The performance of this two-class BCI system has been largely improved, through the exploration of machine learning algorithms [49], [55], [58]-[60], adaptation of both human and machine [61], and extensive subject training protocols [13]. Three major MI studies on 193 [52], 80 [62] and 52 subjects [63], have shown that the proportion of subjects reaching adequate performance (accuracy $\geq 70 \%$ [64]) was approximately $50 \%$ [51]. In the present study, we have shown that the SAO-based two-class BCI can reach an average performance of $85.2 \pm 11.2 \%$ when the discriminative frequency band and the task pair are individually optimized for each subject and only $\sim 10 \%$ of the subjects have an accuracy $<70 \%$. In our previous study we found that the optimal frequency band was quite different across subjects [37], [42], indicating the necessity to do the frequency band selection. These results are in agreement with our previous results [37], where discrimination between SAO-LF with SAO-RT was performed with an average performance of $82.5 \%$ after subject specific frequency band selection. Paired sensory stimulation training will likely provide a way to further improve SAO-based BCI performance. 
With the purpose of getting subjects ready for the task, the vibration burst induced an oscillatory power decrease (ERD) in the [8 26] Hz in both the left and right hemisphere (Fig. 2 (E)), concentrated in the $\mathrm{C} 3$ and $\mathrm{C} 4$ electrodes located above somatosensory cortex (passive tactile stimulation), and in accordance with our previous finding [42]. During the task period (the 3rd to 8th second from the beginning of the trial), ERD/ERS showed a task-related differentiation in the left and right somatosensory hemisphere. In SAO-LF and SAO-RT tasks, the contralateral activation was stronger than that in the ipsilateral side, which is in accordance with our previous results [37], [42]. In contrast, during the SAO-BI task, subjects were instructed to focus the imagined sensation on both hands, resulting in a bilateral activation. The ERD oscillatory activation during SAO-BI was also more pronounced than that during the SAO-ID task, in which subjects were in an idle state and actively suppressed the imagined sensation. This electrophysiological evidence lays the foundation for the current multiple-class SAO-based BCI. Interestingly, the oscillatory dynamic pattern of the four SAO tasks was similar to our previous finding in a tactile BCI, in which four real tactile SS tasks were performed [38], i.e. SS-LF, SS-RT, SS-BI and SS-ID. Generally, the SS showed enhanced ERD activation on both hemispheres due to the passive tactile stimulation. The comparison between SS and SAO among the four tasks will need a future investigation through a within-subject study.

Interestingly, when examining the topographical $\mathrm{R}^{2}$ distribution among different two-class modalities, three-class and four-class modalities, the discriminative power was more concentrated on the right hemisphere. This clearly indicates that the cortical activities of the right hemisphere are modulated to a greater extend by the different SAO tasks. It is possible that a one channel setup using the right hemisphere (C4) channel would have the potential for a practical SAO based BCI system. In current study, all subjects were right-handed. Whether or not the present phenomena of the $\mathrm{R}^{2}$ concentrating on the right hemisphere is caused by handedness would be a question worthy of future investigation.

Independent of any exogenous stimuli, the SAO and MI are cross-modal mental tasks. Covert volitions, both imagined motor intention and imagined sensation intention, are reflected in the spontaneous EEG rhythms. The ERD/ERS analysis has shown that SAO of different hands results in diverse brain activation patterns, with strong ERD activation concentrated on the contralateral hemisphere. Moreover, the $\mathrm{R}^{2}$ discriminative information was mainly concentrated on the left and right hemisphere, further supporting that the brain signals were generated from the sensory-motor cortex. The SAO-induced oscillatory dynamics were similar to those induced by MI, in which the contralateral ERD and ipsilateral ERS were mostly found when subjects performed the hand MI tasks. In our hybrid study of SAO and MI [42], the classification accuracy between SAO-LF and MI-RT was significantly higher than that between MI-LF and MI-RT, and that between SAO-LF and SAO-RT, indicating there was a significant difference between $\mathrm{SAO}$ and MI tasks although ERD/ERS analysis showed similar activations. For better control of the task that the subjects performed, for the SAO experiment, subjects were explicitly instructed to not perform any movement, imagine or attempt movement, but only to feel the sensation of their body. Due to the inherently internal nature of pure SAO mental tasks, we made the instruction clear to maintain subjects on track to perform only the somatosensory attention task rather than a motor task. Moreover, no feedback was provided to the subjects after every trial to avoid any subjective change of mental task strategy. The corresponding activation sources for MI and SAO should be further investigated using simultaneous EEG and fMRI recording, to achieve a greater spatial resolution. Previous fMRI studies have shown that tactile imagery activates the primary somatosensory cortex similar to real tactile stimulation [65], [66]. This supports the feasibility of our proposed sensory imagery paradigm, however the comparison between MI and SAO in terms of brain activation patterns using EEG would be worthy of further investigation.

In the current study, subjects were informed to perform the four imagined sensation tasks according to randomly presented visual cues. The offline analysis results, with regards to the twoclass classification, were comparable with our previous online SAO study [37]. Three-class and Four-class online performance still require further investigation. It is worth noting that SAO is a type of sensory imagery (SI), and SI can also be used when imaging other properties of somatosensory stimulation, such as intensity, type (tactile, temperature, etc.). This property of SI for enhancing system performance would need further investigation.

In this study, subject specific frequency band was selected to evaluate the BCI performance. In our previous study we found that the optimal frequency band differed between subjects [37], [42], indicating the necessity to do the frequency band selection on an individual basis. Interestingly, as shown in Fig. 9 the lower alpha band was not selected at all among the 19 subjects in the two-class, three-class and four-class scenarios, which is in accordance with our previous results [53], indicating a clear difference between lower alpha and upper alpha in BCI performance.

In the current experimental paradigm, a cue-based design was utilized such that the system worked in a synchronous rather than asynchronous mode. Our results indicate the feasibility of separating the somatosensory tasks from the idle state, while a fully asynchronous system based on SAO will require future work. The vibration pulse was primarily used as a cue to allow the subjects to prepare for the subsequent task. In our previous study [37], we demonstrated that the SAO paradigm is capable to operate without any external stimuli, where it should be considered as a truly independent BCI.

\section{CONCLUSION}

We presented and validated a stimulus-independent multipleclass BCI system based on SAO, with a group-level BCI performance of $69.5 \pm 16.2 \%$ for a three-class scenario and $53.3 \pm 17.0 \%$ for a four-class scenario. These multiple-class BCI systems enlarge the types of current multiple-class BCIs. The combination of $\mathrm{MI}$ and SAO for offering more discriminative commands and higher BCI performance will be further investigated in future studies.

\section{ACKNOWLEDGEMENT}

We would like to thank all volunteers in the study. This work is supported by the University Starter Grant of the University 
of Waterloo (No. 203859) and the National Natural Science Foundation of China (Grant No. 51620105002).

\section{REFERENCE}

[1] J. R. Wolpaw, N. Birbaumer, D. J. McFarland, G. Pfurtscheller, T. M. Vaughan, and others, "Brain-computer interfaces for communication and control," Clin. Neurophysiol., vol. 113, no. 6, pp. 767-791, 2002.

[2] C. Guger, H. Ramoser, and G. Pfurtscheller, "Real-time EEG analysis with subject-specific spatial patterns for a brain-computer interface (BCI)," IEEE Trans. Rehabil. Eng., vol. 8, no. 4, pp. 447456, 2000

[3] G. Buzsáki, C. A. Anastassiou, and C. Koch, "The origin of extracellular fields and currents?EEG, ECoG, LFP and spikes," Nat. Rev. Neurosci., vol. 13, no. 6, pp. 407-420, 2012.

[4] G. Santhanam, S. I. Ryu, M. Y. Byron, A. Afshar, and K. V Shenoy, "A high-performance brain--computer interface," Nature, vol. 442, no. 7099, pp. 195-198, 2006.

[5] J. R. Wolpaw et al., "BCI meeting 2005-workshop on signals and recording methods," IEEE Trans. neural Syst. Rehabil. Eng., vol. 14, no. 2, pp. 138-141, 2006.

[6] A. Kübler et al., "Patients with ALS can use sensorimotor rhythms to operate a brain-computer interface," Neurology, vol. 64, no. 10, pp. 1775-1777, 2005.

[7] M. J. Vansteensel et al., "Fully implanted brain--computer interface in a locked-in patient with ALS," N. Engl. J. Med., vol. 375, no. 21 , pp. 2060-2066, 2016.

[8] E. W. Sellers, D. B. Ryan, and C. K. Hauser, "Noninvasive braincomputer interface enables communication after brainstem stroke," Sci. Transl. Med., vol. 6, no. 257, p. 257re7, Oct. 2014.

[9] S. R. Soekadar, N. Birbaumer, M. W. Slutzky, and L. G. Cohen, "Brain--machine interfaces in neurorehabilitation of stroke," Neurobiol. Dis., vol. 83, pp. 172-179, 2015.

[10] N. N. Johnson et al., "Combined rTMS and virtual reality braincomputer interface training for motor recovery after stroke," $J$. Neural Eng., vol. 15, no. 1, 2018.

[11] J. Long, Y. Li, H. Wang, T. Yu, J. Pan, and F. Li, "A Hybrid Brain Computer Interface to Control the Direction and Speed of a Simulated or Real Wheelchair," Neural Syst. Rehabil. Eng. IEEE Trans., vol. 20, no. 5, pp. 720-729, Sep. 2012.

[12] T. Carlson and J. del R. Millan, "Brain-controlled wheelchairs: a robotic architecture," IEEE Robot. Autom. Mag., vol. 20, no. 1, pp. 65-73, 2013.

[13] A. J. Doud, J. P. Lucas, M. T. Pisansky, and B. He, "Continuous three-dimensional control of a virtual helicopter using a motor imagery based brain-computer interface," PLoS One, vol. 6, no. 10, p. e26322, 2011

[14] K. LaFleur, K. Cassady, A. Doud, K. Shades, E. Rogin, and B. He, "Quadcopter control in three-dimensional space using a noninvasive motor imagery-based brain-computer interface.," J. Neural Eng., vol. 10, no. 4, p. 46003, Aug. 2013.

[15] J. Meng, S. Zhang, A. Bekyo, J. Olsoe, B. Baxter, and B. He, "Noninvasive Electroencephalogram Based Control of a Robotic Arm for Reach and Grasp Tasks," Sci. Rep., vol. 6, no. November, p. 38565, 2016.

[16] K. K. Ang and C. Guan, "Brain-computer interface for neurorehabilitation of upper limb after stroke," Proc. IEEE, vol. 103 , no. 6, pp. 944-953, 2015.

[17] F. Pichiorri, N. Mrachacz-Kersting, M. Molinari, S. Kleih, A. Kübler, and D. Mattia, "Brain-computer interface based motor and cognitive rehabilitation after stroke - state of the art, opportunity, and barriers: summary of the BCI Meeting 2016 in Asilomar," Brain-Computer Interfaces, vol. 4, no. 1-2, pp. 53-59, 2017.

[18] S. Silvoni et al., "Brain-Computer Interface in Stroke: A Review of Progress," Clin. EEG Neurosci., vol. 42, no. 4, pp. 245-252, 2011.

[19] A. Ramos-Murguialday et al., "Brain-machine interface in chronic stroke rehabilitation: A controlled study," Ann. Neurol., vol. 74, no. 1, pp. 100-108, 2013.

[20] G. Pfurtscheller, C. Neuper, and others, "Motor imagery activates primary sensorimotor area in humans.," Neurosci. Lett., vol. 239, no. $2-3$, p. 65,1997

[21] G. Pfurtscheller and C. Neuper, "Motor imagery and direct braincomputer communication," Proc. IEEE, vol. 89, no. 7, pp. 1123
1134,2001

[22] B. He, B. Baxter, B. J. Edelman, C. C. Cline, and W. W. Ye, "Noninvasive brain-computer interfaces based on sensorimotor rhythms," Proc. IEEE, vol. 103, no. 6, pp. 907-925, 2015.

[23] N. Sharma, V. M. Pomeroy, and J.-C. Baron, "Motor imagery: a backdoor to the motor system after stroke?," Stroke., vol. 37, no. 7, pp. 1941-52, Jul. 2006.

[24] J. J. Daly and J. R. Wolpaw, "Brain--computer interfaces in neurological rehabilitation," Lancet Neurol., vol. 7, no. 11, pp. 1032-1043, 2008.

[25] G. Pfurtscheller, C. Neuper, D. Flotzinger, and M. Pregenzer, "EEG-based discrimination between imagination of right and left hand movement," Electroencephalogr. Clin. Neurophysiol., vol. 103, no. 6, pp. 642-651, 1997.

[26] B. Graimann, J. E. Huggins, S. P. Levine, and G. Pfurtscheller, "Visualization of significant ERD/ERS patterns in multichannel EEG and ECoG data," Clin. Neurophysiol., vol. 113, no. 1, pp. 4347, 2002.

[27] G. Pfurtscheller, "Spatiotemporal ERD/ERS patterns during voluntary movement and motor imagery," Suppl. Clin. Neurophysiol., vol. 53, pp. 196-198, 2000.

[28] C. Neuper, M. Wörtz, and G. Pfurtscheller, "ERD/ERS patterns reflecting sensorimotor activation and deactivation," Prog. Brain Res., vol. 159, pp. 211-222, 2006.

[29] Y. Hashimoto and J. Ushiba, "EEG-based classification of imaginary left and right foot movements using beta rebound," Clin. Neurophysiol., vol. 124, no. 11, pp. 2153-2160, 2013.

[30] G. Pfurtscheller and F. H. da Silva, "Event-related EEG/MEG synchronization and desynchronization: basic principles," Clin. Neurophysiol., vol. 110, no. 11, pp. 1842-1857, 1999.

[31] E. Houdayer, E. Labyt, F. Cassim, J. L. Bourriez, and P. Derambure, "Relationship between event-related beta synchronization and afferent inputs: analysis of finger movement and peripheral nerve stimulations.," Clin. Neurophysiol., vol. 117, no. 3, pp. 628-36, Mar. 2006.

[32] C. Nangini, B. Ross, F. Tam, and S. J. Graham, "Magnetoencephalographic study of vibrotactile evoked transient and steady-state responses in human somatosensory cortex.," Neuroimage, vol. 33, no. 1, pp. 252-262, Oct. 2006.

[33] M. Severens, J. Farquhar, P. Desain, J. Duysens, and C. Gielen, "Transient and steady-state responses to mechanical stimulation of different fingers reveal interactions based on lateral inhibition," Clin. Neurophysiol., vol. 121, no. 12, pp. 2090-2096, Dec. 2010.

[34] L. Yao, J. Meng, D. Zhang, X. Sheng, and X. Zhu, "Selective Sensation Based Brain-Computer Interface via Mechanical Vibrotactile Stimulation," PLoS One, vol. 8, no. 6, p. e64784, 2013.

[35] L. Yao, J. Meng, D. Zhang, X. Sheng, and X. Zhu, "Combining motor imagery with selective sensation toward a hybrid-modality BCI," IEEE Trans. Biomed. Eng., vol. 61, no. 8, pp. 2304-2312, 2014

[36] L. Yao, J. Meng, X. Sheng, D. Zhang, and X. Zhu, “A Novel Calibration and Task Guidance Framework for Motor Imagery BCI via Tendon Vibration Induced Sensation with Kinesthesia Illusion," J. Neural Eng., vol. 12, no. 1, p. 16005, 2015.

[37] L. Yao, X. Sheng, D. Zhang, N. Jiang, D. Farina, and X. Zhu, "A BCI System based on Somatosensory Attentional Orientation," IEEE Trans. Neural Syst. Rehabil. Eng., vol. 4320, no. c, pp. 1-1, Jan. 2016.

[38] L. Yao et al., "A Multi-Class Tactile Brain-Computer Interface Based on Stimulus-Induced Oscillatory Dynamics," IEEE Trans. Neural Syst. Rehabil. Eng., vol. 26, no. 1, pp. 3-10, Jan. 2018.

[39] G. R. Muller-Putz, R. Scherer, C. Neuper, and G. Pfurtscheller, "Steady-state somatosensory evoked potentials: suitable brain signals for brain-computer interfaces?," Neural Syst. Rehabil. Eng. IEEE Trans., vol. 14, no. 1, pp. 30-37, 2006.

[40] S. Ahn, M. Ahn, H. Cho, S. Chan Jun, and S. C. Jun, "Achieving a hybrid brain-computer interface with tactile selective attention and motor imagery," J. Neural Eng., vol. 11, no. 6, p. 66004, Dec. 2014.

[41] C. Breitwieser, C. Pokorny, R. M. Gernot, and others, "A hybrid three-class brain? computer interface system utilizing SSSEPs and transient ERPs," J. Neural Eng., vol. 13, no. 6, p. 66015, 2016.

[42] L. Yao et al., "A Stimulus-Independent Hybrid BCI Based on Motor Imagery and Somatosensory Attentional Orientation," IEEE Trans. Neural Syst. Rehabil. Eng., vol. 25, no. 9, pp. 1674-1682, 2017.

[43] L. Yao, X. Sheng, N. Mrachacz-Kersting, X. Zhu, D. Farina, and N. 
Jiang, "Decoding covert somatosensory attention by a BCI system calibrated with tactile sensation," IEEE Trans. Biomed. Eng., vol. 9294, no. c, pp. 1-1, 2017.

[44] R. Oostenveld, P. Fries, E. Maris, and J. M. Schoffelen, "FieldTrip: open source software for advanced analysis of MEG, EEG, and invasive electrophysiological data," Comput. Intell. Neurosci., vol. 2011, p. 1, 2011.

[45] T. H. Wonnacott and R. J. Wonnacott, Introductory statistics, vol. 19690. Wiley Chichester, 1990.

[46] G. Schalk, D. J. McFarland, T. Hinterberger, N. Birbaumer, and J. R. Wolpaw, "BCI2000: a general-purpose brain-computer interface (BCI) system," IEEE Trans. Biomed. Eng., vol. 51, no. 6, pp. 10341043, 2004.

[47] H. Ramoser, J. Muller-Gerking, and G. Pfurtscheller, "Optimal spatial filtering of single trial EEG during imagined hand movement," IEEE Trans. Rehabil. Eng., vol. 8, no. 4, pp. 441-446, 2000.

[48] B. Blankertz, R. Tomioka, S. Lemm, M. Kawanabe, and K.-R. Muller, "Optimizing spatial filters for robust EEG single-trial analysis," IEEE Signal Process. Mag., vol. 25, no. 1, pp. 41-56, 2008.

[49] J. Meng, L. Yao, X. Sheng, D. Zhang, and X. Zhu, "Simultaneously optimizing spatial spectral features based on mutual information for EEG classification," IEEE Trans. Biomed. Eng., vol. 62, no. 1, pp. 227-240, 2015.

[50] G. R. Müller-putz, R. Scherer, C. Brunner, R. Leeb, and G. Pfurtscheller, "Better than random? A closer look on BCI results," Int. Jouranl Bioelectromagn., vol. 10, no. 1, pp. 52-55, 2008.

[51] M. Ahn and S. C. Jun, "Performance variation in motor imagery brain-computer interface: A brief review," J. Neurosci. Methods, vol. 243, pp. 103-110, 2015.

[52] C. Guger, G. Edlinger, W. Harkam, I. Niedermayer, and G. Pfurtscheller, "How many people are able to operate an EEG-based brain-computer interface (BCI)?," Neural Syst. Rehabil. Eng. IEEE Trans., vol. 11, no. 2, pp. 145-147, Jun. 2003.

[53] L. Yao, X. Sheng, N. Mrachacz-Kersting, X. Zhu, D. Farina, and N. Jiang, "Performance of Brain-Computer Interfacing Based on Tactile Selective Sensation and Motor Imagery," IEEE Trans. Neural Syst. Rehabil. Eng., vol. 4320, no. c, pp. 1-1, 2017.

[54] G. Pfurtscheller, C. Brunner, A. Schlögl, and F. H. L. Da Silva, "Mu rhythm (de) synchronization and EEG single-trial classification of different motor imagery tasks," Neuroimage, vol. 31, no. 1, pp. 153-159, 2006.

[55] M. Tangermann et al., "Review of the BCI competition IV," Front. Neurosci., vol. 6, 2012.

[56] A. Schlögl, F. Lee, H. Bischof, and G. Pfurtscheller, "Characterization of four-class motor imagery EEG data for the BCI-competition 2005," J. Neural Eng., vol. 2, no. 4, p. L14, 2005.

[57] E. V. C. Friedrich, R. Scherer, and C. Neuper, "Long-term evaluation of a 4-class imagery-based brain-computer interface," Clin. Neurophysiol., vol. 124, no. 5, pp. 916-927, 2013.

[58] B. Blankertz, G. Dornhege, M. Krauledat, K.-R. Müller, and G. Curio, "The non-invasive Berlin Brain-Computer Interface: Fast acquisition of effective performance in untrained subjects," Neuroimage, vol. 37, no. 2, pp. 539-550, 2007.

[59] F. Lotte, M. Congedo, A. Lécuyer, F. Lamarche, B. Arnaldi, and others, "A review of classification algorithms for EEG-based brain-computer interfaces," J. Neural Eng., vol. 4, 2007.

[60] B. Blankertz et al., "The BCI competition III: Validating alternative approaches to actual BCI problems," IEEE Trans. Neural Syst. Rehabil. Eng., vol. 14, no. 2, pp. 153-159, 2006.

[61] C. Vidaurre, C. Sannelli, K.-R. Müller, and B. Blankertz, "Coadaptive calibration to improve BCI efficiency," J. Neural Eng., vol. 8, no. 2, p. 25009, 2011.

[62] B. Blankertz et al., "Neurophysiological predictor of SMR-based BCI performance," Neuroimage, vol. 51, no. 4, pp. 1303-1309, Jul. 2010.

[63] M. Ahn, H. Cho, S. Ahn, and S. C. Jun, "High theta and low alpha powers may be indicative of BCI-illiteracy in motor imagery," PLOS One, vol. 8, no. 11, p. e80886, 2013.

[64] B. Z. Allison, C. Brunner, V. Kaiser, G. R. Müller-Putz, C. Neuper, and G. Pfurtscheller, "Toward a hybrid brain--computer interface based on imagined movement and visual attention," J. Neural Eng., vol. 7, no. 2, p. 26007, 2010.

[65] S. S. Yoo, D. K. Freeman, J. J. McCarthyIII, and F. A. Jolesz, "Neural substrates of tactile imagery: A functional MRI study," Neuroreport, vol. 14, no. 4, pp. 581-585, 2003.

[66] T. T. Schmidt, D. Ostwald, and F. Blankenburg, "Imaging tactile imagery: Changes in brain connectivity support perceptual grounding of mental images in primary sensory cortices," Neuroimage, vol. 98, pp. 216-224, 2014. 